\title{
VELOCITY AFTER-EFFECTS: THE EFFECTS OF ADAPTATION TO MOVING STIMULI ON THE PERCEPTION OF SUBSEQUENTLY SEEN MOVING STIMULI
}

\author{
PETER ThOMPSON* \\ The Psychological Laboratory. Cambridge University, Downing Street. \\ Cambridge, England
}

(Receited 9 March 1980)

\begin{abstract}
Following adaptation to movement in one direction, subsequently seen movement in both the same and opposite directions can be distorted in its apparent velocity.

Experiments in which the adaptation and test stimuli moved in the same direction systematically varied the spatial and temporal parameters of the stimuli. These experiments suggest that the velocity rather than the temporal frequency of the adaptation stimulus determines the magnitude of the velocity alter-effect.

When adaptation stimuli move in the opposite direction to the test stimuli, changes in adaptation velocity or temporal frequency have little effect on the after-effect.
\end{abstract}

\section{INTRODUCTION}

If after looking at a moving pattern for a few seconds you transfer your gaze to a stationary pattern it will appear to be moving in the opposite direction to that of the previously seen movement. This effect, called the movement after-effect (MAE), is one of the most thoroughly researched phenomena of visual perception. Despite such attention, none of the models of the processes underlying the effect is entirely satisfactory. One reason for this is the lack of standardization of the stimuli used to generate the effect.

Any adequate model of the MAE must also be able to explain the perceived velocity of moving patterns after adaptation to movement. Experiments investigating these velocity after-effects are few and far between compared to the multitude of studies on all aspects of the MAE. Both Wohlgemuth (1911) and Gibson (1937) established that the perceived velocity of a stimulus moving at constant velocity decreases with prolonged inspection, a finding confirmed by Goldstein (1959). Carlson (1962) extended these findings. investigating the effects of adaptation to one rate of movement upon a range of velocities in both the same and in the opposite direction to the adaptation motion. As well as confirming the findings of Wohlgemuth, Gibson and Goldstein, Carlson found that if the adaptation velocity was faster and in the same direction as the test velocity, then test velocities were reduced in apparent speed.

Scott et al. (1963) investigated the effects of adaptation to movement upon test velocities in both the same and opposite directions, using both humans and

- Present address: Department of Psychology, University of York. York. England a rhesus monkey as subjects. Their results showed a small but significant increase in the velocity of a test stimulus moving in the opposite direction to the adaptation motion.

Rapoport (1964), in an experiment similar to Carlson's but using rotary movement, also investigated a wider range of test velocities than Scott tet al. When testing in the adaptation direction. velocities equal to or slower than the adaptation velocity appeared reduced in speed. as found by Carlson. Test velocities in the same direction and faster than the adaptation velocity appeared faster after adaptation: this result was suggested but failed to reach significance in Carlson's study. The effects of adapting to movement in one direction upon the perceived velocity of stimuli moving in the opposite direction were inconclusive. but the increase in perceived velocity predicted by Gibson and the ratio model was not found.

Clymer (1973) has also investigated velocity aftereffects, using a matching technique. Clymer found that following adaptation to moving patterns. patterns moving in the same direction were generally decreased in their apparent speed, although with slow adaptation velocities fast test velocities appeared faster after adaptation.

The experiments described in this paper extend the previous work on velocity after-effects and attempt to evaluate these effects in the light of some theoretical predictions.

\section{METHODS}

\section{Definitions}

Any linearly moving repetitive pattern has a velocity which can be defined in terms of the number of degrees of visual angle traversed by a point of that 
pattern in one second. The pattern also has a temporal frequency, the number of periods of the pattern which pass a point in one second, and a spatial frequency defined as the number of periods of the pattern contained within one degree of visual angle.

These properties are related such that:

Velocity $=$ Temporal Frequency Spatial Frequency

$$
(\text { degs } / \mathrm{sec})=(\text { cycles } / \mathrm{sec}),(\text { cycles deg })
$$

Generation of stimuli

The stimuli in all the experiments were one dimensional sinusoidal gratings generated on the screen of an oscilloscope (Telequipment D83) by the technique described by Schade (1956). as modified by Robson and described by Campbell and Green (1965).

In order to produce a grating which drifts in a controlled manner across the screen it is necessary to ensure that each successive frame displayed is initiated at a different point in the modulation cycle. That is, if the $Z$ axis (luminance) modulation is of frequency $A$, then the trigger signal must be of frequency $(A+B)$ in order for the grating to drift at rate $\mathrm{B}$ across the screen. This was achieved by the multiplication method used by Cooper and Robson (1968). Tolhurst (1973) and Tolhurst et al. (1973). This method has been described in detail by Rogers 119761 and Shapley and Rossetto 119:6). The beaty of using sine wave gratings as the stimuli in this research is that it enables tempotal frequency and spatial trequency to be manipulated independently from one another. This allows effects dependent upon velocity to be disentangled from thuse dependent upon temporal frequency.

\section{Experimental procedures}

Perceived velocities of moving gratings were measured by a matching procedure. Two screens were positioned side by side and between them was a small light upon which the subject fixated throughout. To the subject, $114 \mathrm{~cm}$. away from the screens, each had dimensions of $6^{3} \times 4^{\circ}$, separated by $1^{\circ}$. Both screens had a mean luminance of $31.6 \mathrm{cdm}^{-2}$.

Velocity matches were made in the following way, A moving grating was displayed on the left-hand screen, the test screen, whilst on the other, the match screen, was displayed a moving grating of the same spatial frequency but of variable temporal frequency (see Fig. 1). The subject's task was to manipulate the rate of movement of the match grating until it seemed to be moving at the same rate and in the same direction as the test grating.

\section{baseline procedure}
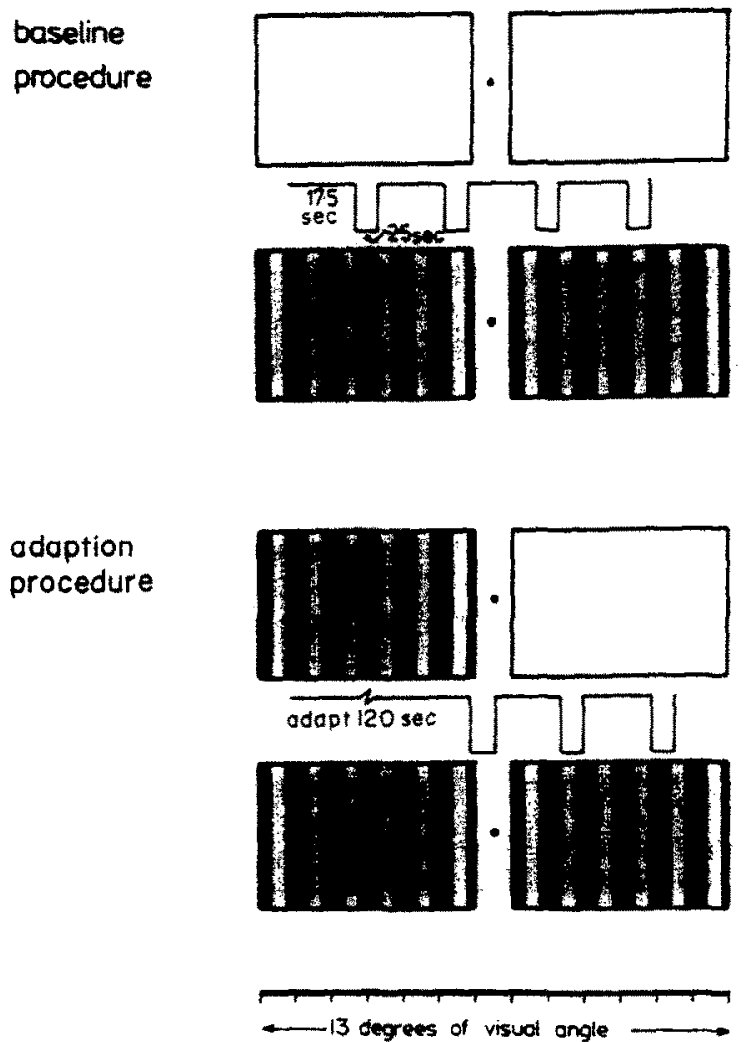

Fig. 1. The baseline and adaptation procedures. In both the baseline and adaptation procedures the upper pair of rectangles represents the screens during the adaptation period and the lower pair the screens during the test period. For convenience gratings are illustrated with square wave rather than sine wave profiles. When no grating was displayed the mean luminance of the screen was unaltered. Although the test and match gratings were always of the same spatial frequency, in certain experiments the adaptation grating was of a different spatial frequency. Further details in the text. 
In any adaptation experiment the matching procedure must be carried out before and immediately after some adaptation stimulus has been presented in order to establish the effects of that adaptation on perceived velocity. If a comparison between pre- and post-adaptation matches is to be made then the procedure in both phases of the experiment should be as similar as possible. It is well known that many effects of adaptation decay very rapidly (e.g. Sekuler and Pantle 1967; Blakemore and Campbell 1969; Blakemore et al., 1970). therefore velocity matches must be made very rapidly. To reduce this problem a "topping up" procedure was adopted which allowed subjects as many attempts as they required to make a suitable match. Following an initial 2 min adaptation period the test and match gratings were presented for $2.5 \mathrm{sec}$. In this time the subject could be expected to make only a very crude match. This was followed by a $17.5 \mathrm{sec}$ topping up period of the adaptation stimulus which was again followed by a $2.5 \mathrm{sec}$ match period. This procedure continued until the subject was satisfied that the match grating appeared to move at the same rate as the test grating. This rate of movement of the match grating was then recorded. In each experiment the presentation order of all test stimuli was randomized.

In the baseline procedure (see Fig. 1) measurements were made in exactly the same way except that the adaptation grating was replaced by a blank screen of the same mean luminance. At least four baseline and four adaptation readings were taken in each condition.

A few preliminary experiments were carried out. One determined that the $2 \mathrm{~min}$ adaptation period, with topping up, was sufficient to achieve a large and stable level of adaptation. A second confirmed that adaptation to a grating on one screen did not affect the appearance of gratings presented on the other screen. The details of these experiments are described elsewhere (Thompson 1976).

Adaptation to a high contrast grating reduces the apparent contrast of subsequently seen suprathreshold gratings (Blakemore et al., 1973). As the perceived rate of movement of a grating is dependent upon its perceived contrast (Thompson, 1976, and in preparation). then adaptation to a moving grating may alter the perceived rate of movement of a subsequently seen grating simply because of this contrast reduction. Preliminary experiments established that adaptation gratings of low contrast $(0.11)$ had very little effect, always less than $3 \mathrm{~dB}$. on the perceived contrast of high contrast $(0.33)$ test gratings. These experiments are described elsewhere (Thompson 1976). Therefore in all the experiments described in this paper the adiuptation gratings had a contrast of 0.11 and the test gratings of 0.33 .

The data in the present paper are not presented in perceived versus real test stimulus movement plots. rather the ordinate, labelled "velocity match" refers to the ratio of the post-adaptation to the baseline vel- ocity matches. A value of 1.0 on this axis represents the condition in which the perceived velocity of the test grating is unaffected by the adaptation grating. values less than 10 mean that adiptation has reduced perceived velocity and values greater than 1.0 mean an increase in perceived velocity.

At least two subjects took part in each experiment and in each case there was good agreement between them.

Although generally not shown on the figures. the standard error associated with each point was calculated. In no case did this error exceed $6^{\circ}$. and the average was about $4^{\circ} ;$. To give an indication of these errors, standard error bars have been plotted in Fig. 2, where the average error is $3.5^{\circ} \%$

\section{Rationale of experiments}

All the experiments followed the same pattern: the effects of some range of adaptation gratings were investigated on some range of test gratings. In any one experiment the spatial frequency, temporal frequency or velocity of adaptation or test gratings was kept constant. The particular conditions examined are represented in Table 1.

In experiment I spatial frequency was held constant at 2 cycles. deg.. so that velocity covaried with temporal frequency. In experiment II the test grating spatial frequency was still held constant, however the adaptation temporal frequency covaried with spatial frequency resulting in a constant adaptation velocity. Experiment III kept velocity constant throughout.

Experiment IV was analogous to experiment I. the only difference being that the adaptation gratings moved in the opposite direction to the test gratings.

\section{EXPERIMENT I}

The velocity after-effects on moving gratings following adaptation to yratings moring in the same direction

Experiment I was designed to examine the effects of adapting to a wide range of velocities upon the subsequent perceived velocities of gratings of the same spatial frequency and moving in the same direction as the adapting motion.

Table 1. A general outline of the adaptation experiments

\begin{tabular}{|c|c|c|}
\hline Experiment & $\begin{array}{l}\text { Adaptation } \\
\text { conditions }\end{array}$ & $\begin{array}{c}\text { Test } \\
\text { conditions }\end{array}$ \\
\hline I & $\begin{array}{l}\text { Spatial } \\
\text { frequency } \\
\text { constant }\end{array}$ & $\begin{array}{l}\text { Spatial } \\
\text { frequency } \\
\text { constant }\end{array}$ \\
\hline II & $\begin{array}{l}\text { Velocity } \\
\text { constant }\end{array}$ & $\begin{array}{l}\text { Spatial } \\
\text { frequency } \\
\text { constant }\end{array}$ \\
\hline III & $\begin{array}{l}\text { Velocity } \\
\text { constant }\end{array}$ & $\begin{array}{l}\text { Velocity } \\
\text { constant }\end{array}$ \\
\hline IV & $\begin{array}{l}\text { Spatial } \\
\text { frequency } \\
\text { constant }\end{array}$ & $\begin{array}{l}\text { Spatial } \\
\text { frequency } \\
\text { constant }\end{array}$ \\
\hline
\end{tabular}




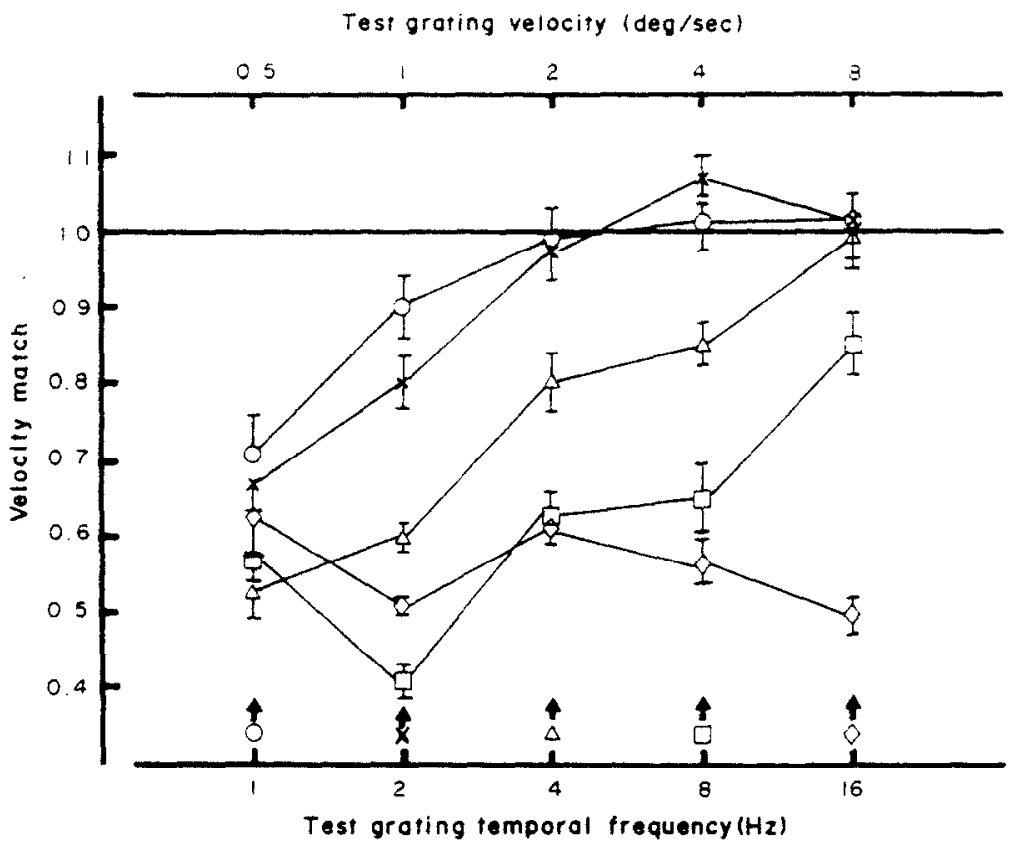

Fig. 2. The effects on perceived velocity of adaptation to movement in the same direction. All gratings at a constant spatial frequency of 2 cycles/deg. Adaptation contrast: 0.11 . Test contrast: 0.33 . Adaptation

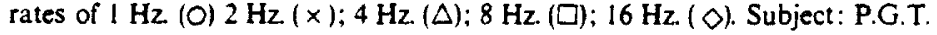

Five different adapting rates of movement were used $(1,2,4,8$ and $16 \mathrm{~Hz})$, and their effects on nine different test rates between $1-16 \mathrm{~Hz}$ were investigated. All gratings in this experiment were of spatial frequency 2 cycles/deg. The experimental procedure has been described in the previous section. The results of the experiment (Fig. 2) are plotted with the post-adaptation velocity match expressed as a fraction of the corresponding pre-adaptation or baseline match.

The most striking features of the data are:

(1) The shifts in apparent velocity are very largecompare them for example with the spatial frequency shifts found by Blakemore and Sutton (1969) which never exceeded $30 \%$.

(2) Low test velocities tend to be reduced by a very wide range of adaptation velocities. Indeed, it appears that a fast adaptation velocity is more effective than a slow one at reducing the apparent velocity of a slow test rate. That is, following adaptation at some velocity all slower velocities appear reduced in speed.

(3) At test velocities greater than the adaptation velocity the effects of adaptation decrease and disappear when the test grating velocity is sufficiently higher than the adaptation velocity.

(4) There is no reliable over-estimation of velocity following adaptation.

\section{EXPERIMENTS II AND III}

Evidence for coding of movement in terms of velocity

All the stimuli in experiment I were of the same spatial frequency, 2 cycles/deg. As a resuit, it is impossible to assess the generality of the after-effects over a range of spatial frequencies. More importantly, it is impossible to distinguish coding of movement in terms of temporal frequency from coding in terms of velocity. That is, if information about movement is coded by a population of units or channels. do these units have some temporal frequency or some vilocity as their preferred "trigger feature" (Barlow 1961)"?

By manipulating the spatial and temporal frequencies of the adaptation and test stimuli we can distinguish between coding by velocity and by temporal frequency. Suppose that the coding of movement is in terms of temporal frequency, then a movernent detector's maximum sensitivity will be to some constant temporal frequency over a range of spatial frequencies. Coding in terms of velocity, on the other hand, demands the greatest sensitivity in the detector at some preferred velocity, regardless of the component spatial and temporal frequencies.

Previous work on the velocity after-effect has cast no light on this problem although some research on the MAE is pertinent. Pantle (1974) examined the magnitude of the MAE, measured both by its duration and by a magnitude estimation technique, and found that it was the temporal frequency of the adaptation stimulus, not its velocity, which determined whether or not the after-effect was maximal.

Data from Moulden (1974) suggest a more complicated relationship. Moulden investigated the magnitude of the MAE generated on a noise field by a range of adaptation velocities. From his experiments Moulden concluded that provided the temporal frequency of the adaptation grating exceeds about $4-5 \mathrm{~Hz}$. the MAE velocity was a function of the adaptation velocity. 
Over et al. (1973) also investigated the MAE magnitude following adaptation to a range of different moving patterns. Their results were not clear cut but suggested that both the spatial frequency and the velocity of the adaptation stimulus contribute to the velocity of the MAE.

A different approach to this problem comes from several threshold studies. Breitmeyer (1973) investigated the detection threshold elevations of stationary gratings of a range of spatial frequencies produced by adaptation to moving wide band noise patterns and also the threshold elevations of moving noise patterns following adaptation to stationary gratings of various spatial frequencies. These experiments suggested that the mechanisms underlying these threshold elevations are most sensitive at temporal frequencies of around $10 \mathrm{~Hz}$.

Tolhurst et al. (1973) came to a qualitatively similar conclusion following a rather different experiment which determined the sensitivity to drifting gratings of different spatial frequencies. For the spatial frequency range investigated $(0.5-1+$ cycles $/ \mathrm{deg})$ the peak sensitivity was about $6 \mathrm{~Hz}$, a result in good agreement with the optimum modulation rate for sensitivity to stationary gratings (Robson. 1966; Kelly 1969).

Further evidence against the analysis of movement in terms of velocity comes from an experiment by Tolhurst (1972). Following adaptation to moving gratings the peak threshold elevation appeared to occur at the adaptation temporal frequency rather than at the adapting velocity.

Although the few studies which have investigated the behaviour of movement channels at detection threshold agree that such mechanisms have preferred temporal frequencies, studies using supra-threshold stimuli (e.g. those investigating MAEs) have produced no such concensus. The purpose of experiments II and III was to ascertain whether those mechanisms underlying the velocity after-effect analyse movement in terms of temporal frequency or velocity.

\section{EXPERIMEYT II}

The dependence of the velocity after-effect on adaptation velocity

Experiment I has shown that the perceived velocity of a 2 cycles deg grating is clearly different following adaptation to a $2 \mathrm{~Hz}, 2$ cycles $/$ deg. (1 deg/sec) grating than after adaptation to an $8 \mathrm{~Hz}, 2$ cycles/deg $14 \mathrm{deg}$; $\mathrm{sec}$ grating. If movement in the human visual system were coded in terms of its temporal frequency then the reason for this difference lies in the difference in adaptation temporal frequencies. If, however, movement is coded in terms of its velocity then the different tuning of the after-effect is caused by the different adaptation velocities.

Analysis by temporal frequency predicts that all adaptation gratings of one temporal frequency will produce qualitatively similar after-effects on a given test grating. Similarly. coding by velocity requires similar effects from adaptation to gratings of the same velocity. More directly the question is this: will the after-effect following adaptation to an $8 \mathrm{~Hz}, 8$ cycles. deg ( 1 deg, 'sec) grating resemble the adaptation produced by a $2 \mathrm{~Hz}$. 2 cycles deg (1 deg sec) grating. as predicted by velocity coding. or that produced by an $8 \mathrm{~Hz}, 2$ cycles deg ( $4 \mathrm{deg}$ sec) grating as predicted by temporal frequency coding?.

Experiment II sought to answer this question by examining the velocity after-effects produced by at range of adaptation gratings on 2 cycles deg test gratings. Adaptation velocities of 1.2 and 4 deg sec were investigated. several adaptation gratings with different spatial and temporal frequencies being used at each velocity. The procedure was identical to that used in the previous experiment.

The results of experiment II are shown in Fig. 3. These results clearly show that the after-effect functions are similar at equal adaptation velocities. This suggests that the mechanisms underlying this aftereffect analyse movement in terms of velocity rather than temporal frequency. This possibility is further tested in experiment III.

\section{EXPERIMEXT III}

\section{Velocity after-effects at constant velocity}

Experiment II has provided considerable evidence that the mechanisms underlying the velocity aftereffect analyse motion in terms of velocity rather than in terms of temporal frequency. If this is so. consider the consequences of keeping the velocity of adaptation and test stimuli constant throughout an experiment. If a range of test stimuli. all of the same velocity, is investigated then following adaptation the shifts in perceived velocity should be of equal magnitude for each test stimulus. The magnitude of this shift may, of course, vary under different adaptation conditions; for example, adaptation to gratings of low temporal frequency may not prove as effective as those of higher temporal frequency.

Experiment III examined the velocity after-effects produced by one grating moving at some velocity upon a range of gratings moving at the same velocity. Three velocities were investigated: 1,2 and $+\mathrm{deg}$, sec. In all other details the experiment was the same as experiment II. The results are shown in Fig. 4. For each test velocity the after-effect magnitude is invariant regardless of the spatial and temporal frequency components of that velocity. This result confirms that velocity after-effects are mediated by a mechanism analysing stimulus velocity rather than stimulus temporal frequency.

\section{EXPERIMENT W}

The effects of adaptation to movement in one direction upon the perceiced rate of motement in the opposite direction

If the human visual system contains direction selective mechanisms then the method of selective adap- 

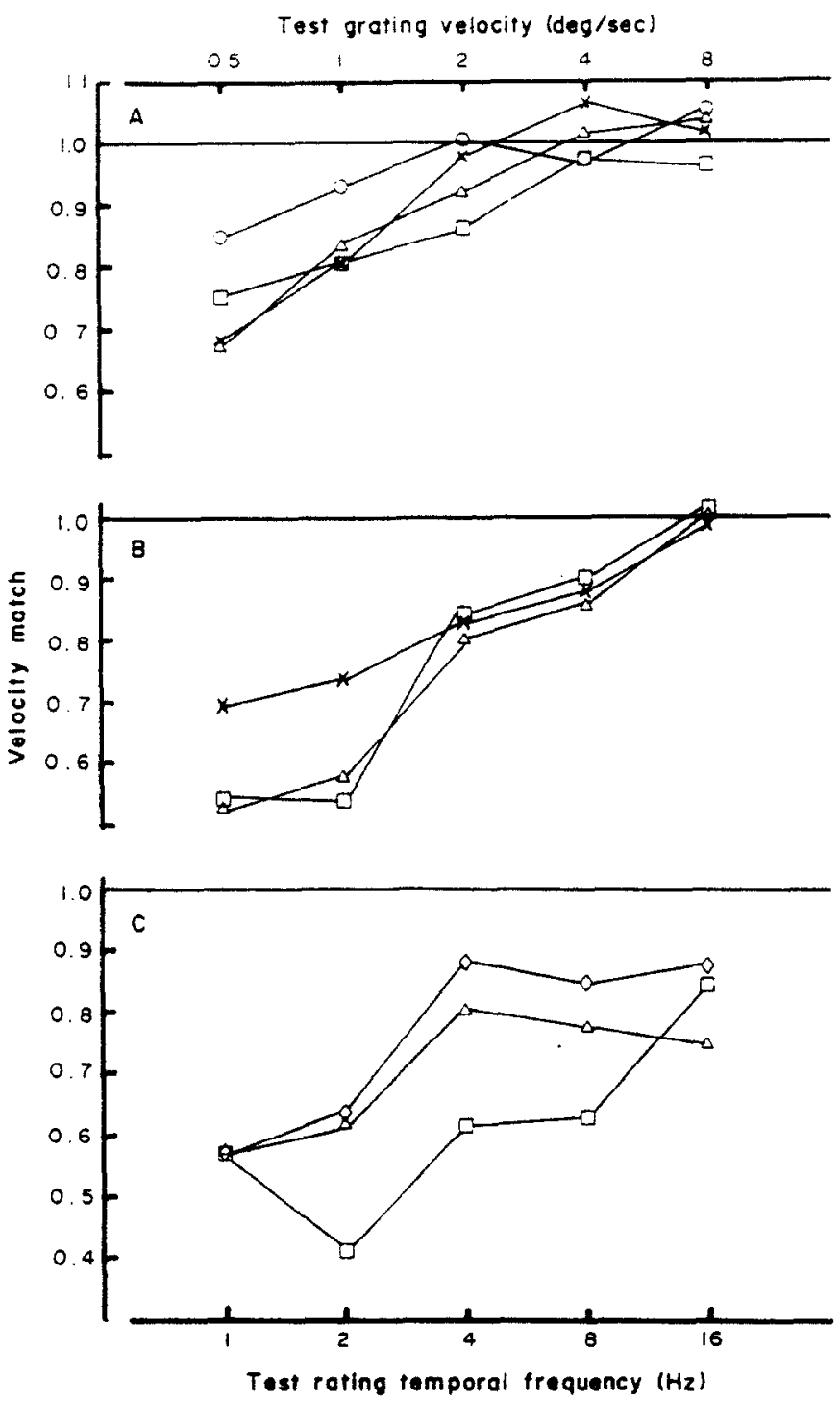

Fig. 3. The effects on perceived velocity of adaptation to movement in the same direction. Test gratings at a constant spatial frequency of 2 cycles/deg. Adaptation contrast: 0.11 . Test contrast: 0.33 . (A) Adaptation gratings at constant velocity of $1 \mathrm{deg} / \mathrm{sec} / \mathrm{Hz}, 1 \mathrm{cycle} / \mathrm{deg}(\mathrm{O}) ; 2 \mathrm{~Hz}, 2 \mathrm{cycles} / \mathrm{deg}(\mathrm{x}): 4 \mathrm{~Hz}, 4$ cycles $/ \mathrm{deg}(\triangle) ; 8 \mathrm{~Hz}, 8 \mathrm{cycles} / \mathrm{deg}(\square)$. (B) Adaptation gratings at constant velocity of $2 \mathrm{deg} / \mathrm{sec} .2 \mathrm{~Hz}$, 1 cycle/deg. $(x) ; 4 \mathrm{~Hz}, 2$ cycles/deg $(\Delta) ; 8 \mathrm{~Hz}, 4$ cycles/deg (D). (C) Adaptation gratings at constant velocity of $4 \mathrm{deg} / \mathrm{sec}$. $4 \mathrm{~Hz}, 1$ cycle/deg. $(\Delta) ; 8 \mathrm{~Hz}, 2$ cycles/deg (D); $16 \mathrm{~Hz}, 4 \mathrm{cycles} / \mathrm{deg}$ ( $O$ ) Subject:

P.G.T.

tation is well suited to investigate them. Sekuler and Ganz (1963), Clarke (1974), Pantle and Sekuler (1969) and Tolhurst (1973) have provided evidence which has established that at least some movement channels are direction selective, but that there is some threshold elevation experienced for gratings drifting in one direction following adaptation to movement in the opposite direction. If there are direction selective channels in the human visual system the question arises of whether channels for opposite directions are independent of one another. Levinson and Sekuler (1975) found no evidence of subthreshold summation between oppositely moving gratings, which suggests the independence of channels tuned for opposite directions of motion, at threshold at least. Sekuler
(1975) has suggested that this independence at and below threshold "is quite different from what would be expected if detection were based on a ratio of responses in mechanisms tuned to opposite directions of motion." More recently Watson. et al. (1980) have modified this view, showing that at slow velocities there is almost total summation between opposite directions of movement at detection threshold.

There is general agreement in all the published studies that following adaptation to a moving pattern the perceived velocity of patterns moving in the opposite direction is affected. There is, however. little agreement on the nature of this change, almost certainly because the lack of a standard stimulus has made comparisons between studies impossible. This 
general confusion prompted an experiment to investigate the effects of adaptation to gratings moving in one direction upon the perceived velocity of test gratings moving in the opposite direction.

Experiment IV was identical in procedure to experiment I with the single exception that the direction of movement of the adaptation grating was reversed. That is, velocity matches to rightward moving gratings were made before and after a leftward moving adaptation grating had been presented. The results are shown in Fig. 5 .

The important features of the resuits are these:

(1) The velocity shifts are generally smaller than those obtained when adaptation and test gratings move in the same direction.

(2) At low test rates, velocity is over-estimated. That is. the shift in velocity is in the direction predicted by the ratio model.

(3) At intermediate rates of test movement, adaptation in the opposite direction leads to a small but consistent under-estimation of velocity.

\section{DISCLSSION}

The findings of these experiments can be compared with the results of previous studies of velocity aftereffects:

(1) The well established finding that a moving pattern appears to be moving slower after prolonged inspection. that is adapting and testing at the same velocity (Wohlgemuth, 1911: Gibson 1937: Goldstein. 1959; Carlson 1962; Scott et al., 1963: Rapoport

Test groting spatial frequency (cycles/deg)

$A$
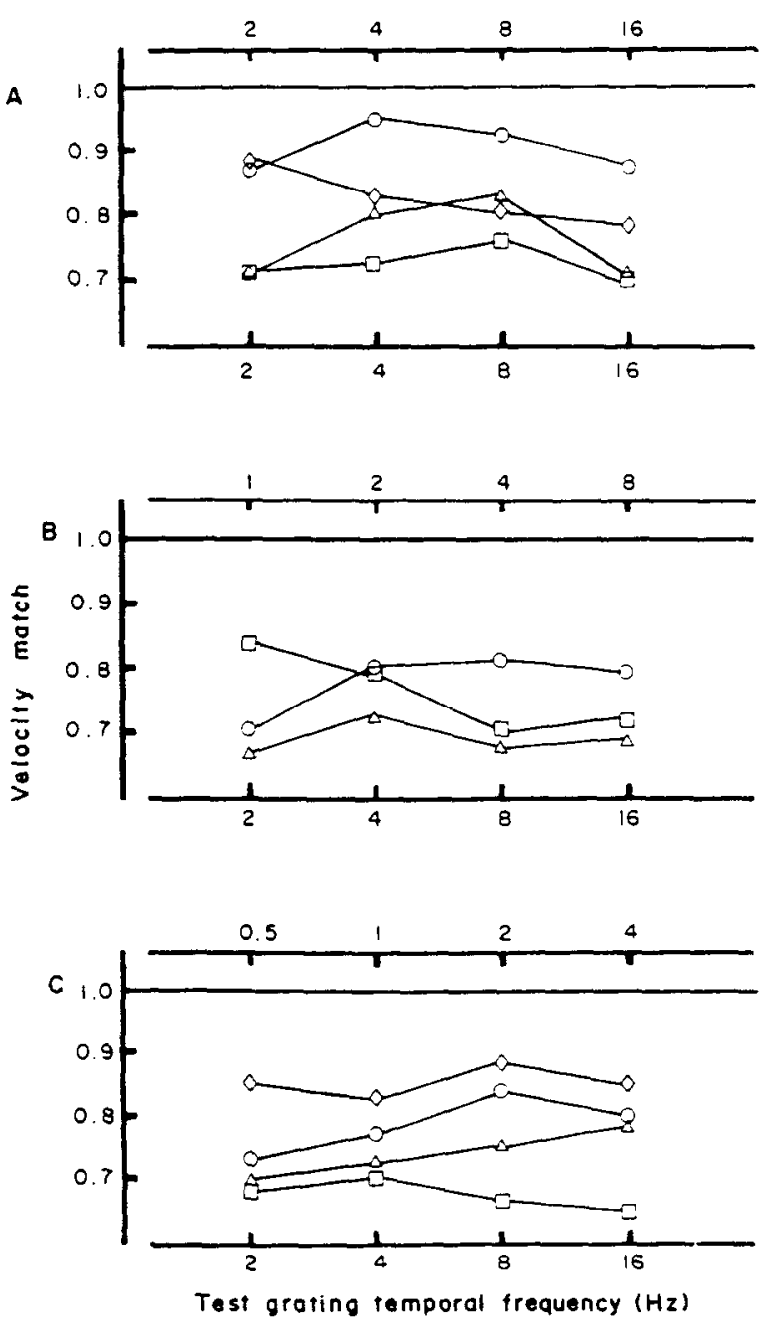

Fig 4. The effects on perceived velocity of adaptation to movement in the same direction. Adaptation contrast: 0.11 . Test contrast: 0.33 . (A) All gratings at constant velocity of $I \mathrm{deg} / \mathrm{sec}$. Adaptation stimuli of $1 \mathrm{~Hz}, 1$ cycle/deg $(O) ; 2 \mathrm{~Hz}, 2$ cycles $/ \operatorname{deg}(\Delta) ; 4 \mathrm{~Hz}, 4$ cycles $/ \operatorname{deg}(\square) ; 8 \mathrm{~Hz}, 8$ cycles deg $(\diamond)$. (B) All gratings at constant velocity of $2 \mathrm{deg} / \mathrm{sec}$. Adaptation stimuli of $2 \mathrm{~Hz}, 1$ cycle/deg $(0) ; 4 \mathrm{~Hz} 2 \mathrm{cycles} / \mathrm{deg}$ $(\triangle) ; 8 \mathrm{~Hz}, 4 \mathrm{cycles} / \mathrm{deg}(\square)$. (C) All gratings at constant velocity of $4 \mathrm{deg} / \mathrm{sec}$. Adaptation stimuli of $2 \mathrm{~Hz}$. 0.5 cycles $/ \mathrm{deg}(0) ; 4 \mathrm{~Hz}, 1$ cycle $/ \operatorname{deg}(\Delta) ; 8 \mathrm{~Hz}, 2$ cycles $/ \operatorname{deg}(\square) ; 16 \mathrm{~Hz}, 4$ cycles $/ \mathrm{deg}(O)$. Subject: P.G.T. 


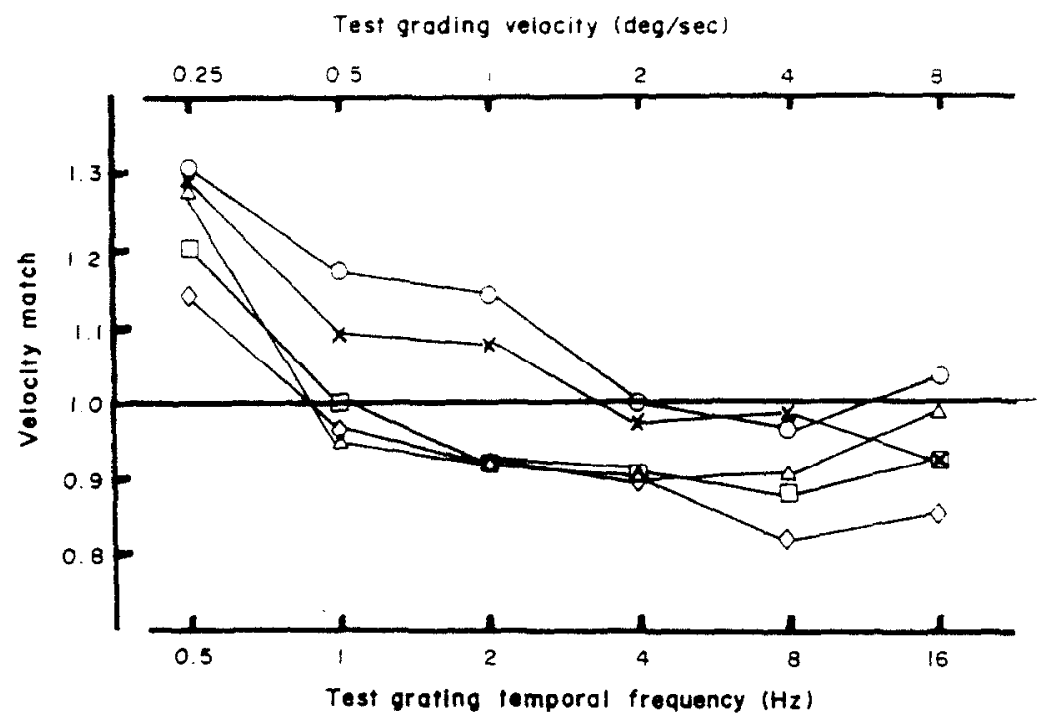

Fig. 5. The effects on perceived velocity of adaptation to movement in the opposite direction. All gratings at constant spatial frequency of 2 cycles/deg. Adaptation contrast: 0.11 . Test contrast: 0.33 . Adaptation rates of $1 \mathrm{~Hz}(0) ; 2 \mathrm{~Hz}(x) ; 4 \mathrm{~Hz}(\Delta) ; 8 \mathrm{~Hz}(\square) ; 16 \mathrm{~Hz}(0)$. Subject: P.G.T.

1964; Clymer, 1973) is replicated and shown to be true over a wide range of velocities.

(2) Test velocities slower than and in the same direction as the adaptation velocity were always found to be reduced after adaptation, again a result found by all those who have investigated this condition (Carlson, 1962; Scott et al., 1963; Rapoport, 1964; Clymer, 1973).

(3) The perceived velocity of test gratings moving faster than, and in the same direction as the adaptation grating was decreased or unchanged after adaptation.

(4) Most previous studies have found that test gratings moving faster than and in the same direction as the adaptation grating tend to appear increased in their velocity after adaptation. This discrepancy between previous reports and the results presented here can be explained. Blakemore et al. (1973) have shown that adapting to a high contrast grating reduces the apparent contrast of subsequently seen gratings. Thompson (1976 and in preparation) has shown that the perceived rate of movement of a grating is dependent upon its apparent contrast. In particular at high temporal rates of movement a reduction in contrast leads to an overestimation of velocity. Therefore previous reports of velocity overestimation following adaptation may merely reflect the results of the adaptation pattern reducing the apparent contrast of the test figure. In all the experiments reported in this paper adaptation gratings of low contrast $(0.11)$ and test gratings of high contrast $(0.33)$ were used. This ensured that the test grating contrast was never reduced by the adaptation grating by more than $3 \mathrm{~dB}-$ a reduction that produces less than a $10 \%$ shift in perceived velocity.

These results allow certain conclusions to be drawn. In a single direction more than a single chan- nel appears to be involved in the processing of velocity, because of the "differential speed tuning" (Clymer, 1973) found in experiment 1. That is. on any single channel model the velocity shifts found after adaptation should reflect the shape of that single channel. Different adaptation stimuli might affect the single channel to different degrees, but they could not produce the differently shaped after-effect functions actually found.

Furthermore the channels involved in the mediation of the after-effect appear to be velocity channels rather than temporal frequency channels in the sense that for a given range of test stimuli a similar pattern of after-effects is found when adaptation stimuli at constant velocity are employed, but not when either their spatial or temporal frequency alone is held constant.

This conclusion, however, should be regarded with some caution. Whilst it does accurately describe the results of this study it is quite possible that some combination of spatial frequency channels with suitable temporal response could produce the same results.

Acknowledgements-This paper reports some of the experiments in my doctoral thesis. I thank Ol Braddick who supervised this work, David Tolhurst for his advice and help, and Jack Nachmias for helpful criticisms of an earlier draft of this paper. I thank also all those members of the Craik Laboratory, Cambridge, who, knowingly and unknowingly provided the apparatus for this research, in particular Colin Blakemore (MRC grant G972/463/B). Throughout my work the technical heip of Phil Taylor and Leslie Wynn was invaluable. I was supported by an SSRC research studentship.

\section{REFERENCES}

Barlow H. B. (1961) The coding of sensory messages. In Current Problems in Animal Behaviour (Edited by 
Thorpe W. H. and Zangwill O. L.), pp. 331-360. Cam. bridge Univ. Press.

Blakemore C. and Campbell F. W. (1969) On the existence of neurones in the human visual system selectively sensitive to the arientation and size of retinal images. J. Phy siol. 203. 237-260.

Blakemore C. Muncey J. P. J. and Ridley R. M. (1973) Stimulus specificity in the human visual system. Fision Res. 13.1915-19:31.

Blakemore C., Nachmias J. and Sutton P. 1970) The perceived spatial frequency shift: evidence for frequency. selective neurones in the human brain. J. Physiol. 210. $737-750$.

Blakemure C. and Sutton P. (1969) Size adaptation: a new alter-effect. Science. YY $166.245-247$.

Breitmever B. $(1973)$ A relationship between the detection of size, rate, orientation and direction of motion in the human visual system. Lision Res. 13, $+1-58$.

Campbell F. W. and Green D. G. (1965) Optical and retinal factors affecting visual resolution. J. Physiol. 181 $576-593$

Carlson V. R. (1962) Adaptation in the perception of visual velocity. J. Exp. Psychol 64. 192-197.

Clarke P. G. H. (1974) Are visual evoked potentials to motion reversal produced by direction-sensitive brain mechanisms? Vision Res. 14, 1281-1284.

Clymer A. B. (1973) The effect of seen motion on the apparent speed of subsequent test velocities: speed tuning of movement A.Es. Ph.D. Thesis, Columbia Lniversity. Wy.

Cooper G. F. and Robson J. O. 119681 Successive transforms of spatial information in the visual system. I.E.E.E. Conference publication 42

Gibson J. J. (1937) Adaptation with negative aftereffect. Psychol. Ret. 44. 223-244.

Goidstein A. G. (1959) Judgements of visual velocity as a function of length of observation. J. exp. Pisichol. 54. $+57-+61$.

Kelly D. H. 11969). Flickering patterns and lateral inhi. bition. J.O.S.A. 59. $1361-1370$.

Levinson E. and Sekulet R. 1975) The independence of channels in human vision selective for direction of move. ment. J. Physiol. 250, 247-266.

Moulden B. P. (1974) Aftereffects, or at treatise of the consequences of adaptation to movement and tilt. Ph.D. thesis. University of Reading. England.

Over R., Broerse J.. Crassini B. and Lovegrove W. (1973) Spatial determinants of the aftereffect of seen motion. Iision Res. 13. 16\$1-1890.
Pantle A. (1974) Motion aftereffect magnitude as a measure of the spatio-temporal response properties of direction sensitive analysers. Vision Res. 14, 1229-1236.

Pantle A. and Sekuler R. $(1969)$ Contrast response of human visual mechanisms sensitive to orientation and direction of motion. Vision Res. 9. 397-406.

Rapoport J. (1964) Adaptation in the perception of rotary motion. J. exp. Psychol. 67. 26:-267.

Robson J. G. (1966) Spatial and temporal contrast sensitivity functions of the visual system. J.OS.A. $\mathbf{5 6 .}$ $11+1-11+2$

Rogers B. $(1976)$ A technique for generating moving stimuli on a C.R.O. Vision Res. 16. $415-41$ ?

Schade O. H. (1956) Optical and photoelectric analog of the eye. J.O.S.A +6. 721-739.

Scott T. R.. Jordan A. E. and Pow ell D. A. (1963) Does the visual after-effect of motion add algebraically to objeclive motion of the test stimulus? J. exp. Pstchol. 66, $500-505$.

Sekuler R. (1975) Visual motion perception. In Handhook of Perception, Vol. $V$ (Edited by Carterette E.C. and Friedman M. P.) Academic Press. New York.

Sekuler R. and Ganz L. (1963) A new aftereffect of seen motion with a stabilized reunal image, Science 139. $\$ 19-420$.

Sekuler R. and Pantle A. $(1967)$ A model for the aftereffects of seen movement. Vision Res. 7, 427-439.

Shaptey R. M. and Rossetto .f. (1976) An electronic visual stimulator. Befhat. Res. Meth. Instr. 8. 15-20

Thompson P. G. $(1976)$ Velocity after-effects and the perception of movement. Ph.D. thesis, University of Cambridge. England.

Tolhurst D. J. (1972) Sustained and transient detectors in the human visual system. Fellowship dissertation for Churchill College. Cambridge. England.

Tolhurst D. 3. 1973) Separate channels for the analysis of the shape and the movement of a moving visual stimulus. J. Physiol. 231. $385-402$

Tolhurst D. J. Sharpe C. R. and Hart G. (1973) The analysis of the drift rate of moving sinusoidal gratings. Vision Res. 13. 25+5-2555

Watson A. B. Thompson P. G.. Murphy B. J. and Nach. mias $J$. (1980) Summation and discrimination of gratings moving in opposite directions. Vision Res 20. 341347.

Wohlgemuth $A$. $(1911)$ On the after-effect of seen movement. Br. J. Psichol. Monoyr. Suppl. 1, 1-11?. 